\section{Brain, Behavior and Evolution}

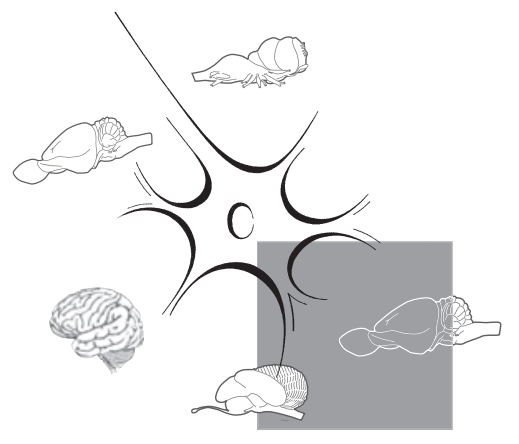

Neuroscience has a diversity problem. Or rather, in addition to the diversity problems that plague academia at large [Hurtado et al., 2010; Shaw, and Stanton, 2012], neuroscience has its own diversity problem. Up until the end of the twentieth century, the number of animal species with which neuroscience research was conducted gradually dwindled until the vast majority of neuroscience used just a handful of species [Manger et al., 2008]. Among "higher" vertebrates (amniotes), house mice (Mus musculus), Norway rats (Rattus norvegicus), and humans (Homo sapiens) were the study systems of choice for answering whatever burning question was keeping any particular neuroscientist awake at night. The common marmoset (Callithrix jacchus) and rhesus macaque (Macaca mulatta) earn honourable mentions, but hardly broaden the phylogenetic breadth of model organisms in neuroscience. These 5 species fall into just 2 Orders (rodents and pri-

The author won the "Thomas Karger Award for Excellence in Evolutionary Neuroscience." The award was presented at the 2017 J.B. Johnston Club meeting on November 10, 2017.

\title{
The Secret Caverns of the Dragon's Brain: Current and Potential Contributions of Lizards to Evolutionary Neuroscience
}

\author{
Daniel Hoops \\ Department of Psychiatry, Douglas Mental Health University Institute, McGill University, \\ Montreal, QC, Canada
}

mates) and the same Class (mammals), leaving the vast majority of the amniote tree of life (phylogeny) unrepresented.

Having tens of thousands of scientists all working on the same model organism does have its advantages. The level of sophistication and precision with which we can manipulate and measure the nervous systems of mice at various levels (e.g., genetic, epigenetic, chemical, cellular, electrophysiological, etc. all the way up to behavioural and social) would have been impossible to achieve had we all been working on different species. Continuing to delve deep and learn absolutely everything we can about the nervous systems of model organisms is essential both for basic neuroscience and to improve the health and wellbeing of human society. However, if we limit our scope to model species, I think we may miss most of the picture.

From talking to prominent neuroscientists and based on my reading of the litera- ture, I believe that the 21st century may be starting to see a reversal of this trend by increasing species diversity in neuroscience, particularly amongst mammals and birds. By broadening our scope within mammals, neuroscience can ask more sophisticated questions that directly build upon what is known in model rodents and primates. By branching into birds, we can learn how the nervous system can achieve the same outcomes using different neural structures and processes, and thus teach us about the universal laws underlying functions that hold great interest for humans, such as vocal learning.

There are 3 major clades of amniotes: birds, mammals, and squamates (lizards and snakes). ${ }^{1}$ Unlike the former 2 clades, squamates do not seem to be experiencing the same renewed interest, despite the work of a few talented investigators worldwide. In 1978, Paul MacLean introduced an edited volume on the behaviour and neurology of lizards by saying "those who are familiar

\section{KARGER}

(c) 2018 S. Karger AG, Basel
1 Those who lament the underappreciation of non-amniotes and the smaller amniote clades in neuro-
science are certainly justified in their criticism and may take issue with my limited focus here. Though
these groups also deserve additional attention and have much untapped potential in neuroscience, I do
not believe this diminishes the importance of the current and potential contributions of squamates. 
with lizards realize that there are more reasons for conducting research on these animals than there are investigators prepared to do the necessary work" [Greenberg, and MacLean, 1978]. This is even more true today.

Squamates are ideal for comparative evolutionary neuroscience because many interesting phenotypes that evolved early in mammals and birds have evolved only recently in some squamate clades. This was a prominent selection criterion when my doctoral supervisor Prof. Scott Keogh recommended I examine brain evolution across the genus Ctenophorus - a group of Australian agamid lizards, referred to colloquially as "dragons." Within this radiation several traits have appeared multiple times near the tips of the phylogeny, allowing for multiple comparisons between closely related species that do and do not have a particular trait of interest.

Because of the neglected status of squamates in neuroscience research, the methods necessary to study their brains are underdeveloped. The past 20 years have seen some significant advances, but many of these publications have received little attention. To develop the resources and methods required for my project, I found and reviewed every peer-reviewed publication on the topic from that period, resulting in a complete overview of the current state of lizard neurobiology. I highlighted what is unique about lizard brains, identified gaps in our understanding, and provided a framework for future studies that included exciting unanswered questions about lizard brain evolution [Hoops, 2016].

One of the most commonly used techniques in neuroscience is perfusion, but a perfusion method had never been published for lizards. I found that the standard mammalian perfusion protocol, which I had used for years working with rats and mice, failed in lizards. Several differences in anatomy can account for this; for example, lizards have 1 ventricle, and though it is partially divided this did not prove to be enough to prevent the backflow of perfusate in my lizards. Furthermore, lizards have not 1 but 3 aortas, only 1 of which sends blood to the brain. Through trial and error and with expert advice I developed a perfusion protocol suitable for lizards. Although perfusing lizards is not novel, the absence of a publicly available protocol represented another way in which squamates are less accessible than other amniotes for neuroscience research. In publishing my perfu- sion protocol [Hoops, 2015], I hoped to help reverse this trend. I encourage others working with understudied groups to consider publishing their protocols, and there are now several journals dedicated to such publications, including MethodsX, Nature Protocols, Bio-Protocol, and BMC Research Notes. Methods can also be uploaded to the Protocol Exchange, where they are assigned a digital object identifier (DOI) and are citable, but are not peer-reviewed.

I examined which of the 2 models of brain evolution, concerted and mosaic, better explained the evolution of the dragon brain. Rather than favouring 1 model, I found evidence for both mosaic and concerted brain evolution in dragons [Hoops et al., 2017a]. Brains showed a pattern of concerted brain evolution with respect to body size and a pattern of mosaic brain evolution with respect to ecology. I believe this is the first demonstration of both the mosaic and concerted models simultaneously influencing the evolution of the same brain regions. Importantly, I demonstrated that the prominence of concerted versus mosaic evolution may depend more on the specific selective pressures being examined rather than the taxonomic group.

While reviewing the literature on brain evolution, I found that the degree of sexual dimorphism in brain structure varies between species, seemingly at random. To determine if there is a pattern underlying interspecific variation in brain sexual dimorphism, I compared the brains of dragon species under strong and weak sexual selection [Hoops et al., 2017b]. Species which were estimated to be under strong sexual selection had more sexually dimorphic brains, and these sexual dimorphisms were limited to brain structures directly involved in controlling sexual behaviour. Therefore, I was able to show that there may be predictable evolutionary explanations underlying the seemingly random variation in brain sexual dimorphism observed in the literature.

Finally, in order to understand how evolution has shaped different brain regions, it is essential to understand the developmental genetic patterning and segmentation that produces those regions. Our classical understanding of how the brain is subdivided is being supplanted by the neuromeric/prosomeric model, which subdivides the brain according to its natural intrinsic boundaries as they are currently understood. However, the boundaries between subdivisions remain blurred, and it is still unclear exactly how many subdivisions exist. Part of the reason is a lack of data across different amniote lineages, because without comparative data it can be difficult to differentiate a fundamental neural subdivision from a recently derived sub-subdivision unique to a particular group. The study of neuromeric divisions in squamates is critical to overcoming such challenges [Desfilis et al., 2017]. Thus, I collaborated with experts in the neuromeric model to identify and delineate the neuromeric divisions of the dragon brain.

As I hope I have demonstrated, squamates are not only important for "bridging the gap" between birds and mammals, but in fact provide an ideal system in which to ask long-standing questions in neuroscience. They are the most suitable amniote group in which to study the evolution of sociality and social cognition due to the relatively recent, and repeated, evolution of social living in squamates [Whiting and While, 2017]. They are ideal for the study of sociosexual behaviours such as courtship behaviour, territoriality, and male-male interactions [Korzan and Summers, 2007]. Also in the realm of sex, they are important for decoupling sex and genetics, since squamate species may have genetic- or temperature-dependent sex determination, or a combination thereof [Holleley et al., 2015]. Squamates continue to be important for the study of adult neurogenesis [Kaslin et al., 2008], due to their abundant production of new neurons throughout life, and of evolutionary developmental neuroscience [Nomura et al., 2013]. Finally, they hold great potential for the study of the impacts of climate change on neural development, cognition, and behaviour [Amiel et al., 2017]. This is just a short list of possibilities; I am sure numerous other questions could be asked.

In my opinion, the main obstacle to future discoveries in neuroscience using squamate model systems is the high barrier to entry, in terms of knowledge and specimen availability. I think it is essential to build collections of reptile brain specimens [Iwaniuk, 2010]. This could take many forms, such as serial stained sections, which could be digitized, uploaded to an online database, and the physical specimens stored in a location accessible to visiting scholars. An intriguing possibility is the creation of a digital library of brains using 3D imaging technologies like enhanced computed tomography and magnetic resonance imaging. By embracing novel systems, including squamates, we can gain in- 
sights into brain function and evolution that would otherwise have been difficult, if not impossible, to achieve. Removing and reducing barriers to this research will be essential for growing and promoting evolutionary neuroscience and demonstrating how this field can contribute to the betterment of humanity.

\section{References}

Amiel JJ, Bao S, Shine R (2017): The effects of incubation temperature on the development of the cortical forebrain in a lizard. Anim Cogn 20:117-125.

-Desfilis E, Abellán A, Sentandreu V, Medina LM (2017): Expression of regulatory genes in the embryonic brain of a lizard and implications for understanding pallial organization and evolution. J Comp Neurol 515:465-492.

Greenberg N, MacLean PD (1978): Behavior and Neurology of Lizards: An Interdisciplinary Colloquium. Rockville, National Institutes of Health.

Holleley CE, O’Meally D, Sarre SD, Graves JAM, Ezaz T, Matsubara K, Azad B, Zhang X, Georges A (2015): Sex reversal triggers the rapid transition from genetic to temperaturedependent sex. Nature 523:79-82.

Hoops D (2015): A perfusion protocol for lizards, including a method for brain removal. MethodsX 2:165-173.

\section{Acknowledgements}

I am grateful to Prof. Scott Keogh and Rose O'Dea for their critical and insightful comments on this article. I am funded by a postdoctoral fellowship from the Quebec Nature and Technology Research Fund (No. 208332).

\section{Disclosure Statement}

The author declares no conflicts of interest.
Hoops D (2016): Structure and function of the lizard and snake brain: recent advances and shifting perspectives; in: Structure and Evolution of Dragon Brains; doctoral thesis, The Australian National University, Canberra.

Hoops D, Ullmann JFP, Janke AL, Vidal-García M, Stait Gardner T, Dwihapsari Y, et al (2017b): Sexual selection predicts brain structure in dragon lizards. J Evol Biol 30:244-256.

Hoops D, Vidal-García M, Ullmann JFP, Janke AL, Stait-Gardner T, Duchêne DA, et al (2017a): Evidence for concerted and mosaic brain evolution in dragon lizards. Brain Behav Evol 90:211-223.

Hurtado S, Newman CB, Tran MC, Chang MJ (2010): Improving the rate of success for underrepresented racial minorities in STEM fields: insights from a national project. New Dir Inst Res 2010:5-15.

Iwaniuk AN (2010): Comparative brain collections are an indispensable resource for evolutionary neurobiology. Brain Behav Evol 76:87-88.

Kaslin J, Ganz J, Brand M (2008): Proliferation, neurogenesis and regeneration in the nonmammalian vertebrate brain. Phil Trans $\mathrm{R}$ Soc B 363:101-122.
Korzan WJ, Summers CH (2007): Behavioral diversity and neurochemical plasticity: selection of stress coping strategies that define social status. Brain Behav Evol 70:257-266.

- Manger PR, Cort J, Ebrahim N, Goodman A, Henning J, Karolia M, et al. (2008): Is 21st century neuroscience too focussed on the rat/ mouse model of brain function and dysfunction? Front Neuroanat 2:5.

Nomura T, Kawaguchi M, Ono K, Murakami Y (2013): Reptiles: a new model for brain evodevo research. J Exp Zool Mol Dev Evol 320:57-73.

Shaw AK, Stanton DE (2012): Leaks in the pipeline: separating demographic inertia from ongoing gender differences in academia. Proc $\mathrm{R}$ Soc B 279:3736-3741.

Whiting MJ, While GM (2017): Sociality in lizards; in Rubenstein DR, Abbot P (eds): Comparative Social Evolution. Cambridge, Cambridge University Press, pp 390-426. 